\title{
Tres especies nuevas de Cyathea (Cyatheales: Cyatheaceae) para Colombia
}

\author{
Three new species of Cyathea (Cyatheales: Cyatheaceae) \\ from Colombia
}

\author{
Alexander Francisco Rojas-Alvarado ${ }^{1}$, Adrian Tejedor ${ }^{2}$
}

\begin{abstract}
Resumen
Se describen e ilustran tres especies nuevas de Cyathea Sm. para Colombia. Cyathea antioquiensis difiere de $C$. latevagans por sus escamas del rizoma con un margen pálido más delgado, pecíolo y raquis con caspilla y pinnas sésiles linear-oblongas. Cyathea incognita difiere de C. gracilis por su hábitat terrestre, rizoma más delgado, escamas del rizoma con un ancho margen pálido, frondas más cortas, pecíolo más delgado, lámina 2-pinnada, raquis atropurpúreo, pinnas sésiles y soros sin indusio. Cyathea longipetiolulata difiere de $C$. divergens por sus frondas ascendentes, pecíolo y raquis sin caspa de escuámulas y pelos, pinnas y pínnulas con pedículos más largos, menos segmentos por pínnula e indusio evanescente.
\end{abstract}

Palabras clave: Cyathea antioquiensis, Cyathea incognita, Cyathea longipetiolulata, Helechos Arborescentes, Neotrópico.

\begin{abstract}
Three new species of Cyathea Sm. are described and illustrated from Colombia. Cyathea antioquiensis differs from $C$. latevagans by its rhizome scales with a narrower pale margin, stipe and rachis with scurf and sessile linear-oblong pinnae. Cyathea incognita differs from C. gracilis by its terrestrial habit, thinner rhizome, rhizome scales with a broad pale margin, shorter fronds, thinner stipe, bipinnate blade, atropurpureous rachis, sessile pinnae and sori without indusia. Cyathea longipetiolulata differ from C. divergens by its ascending fronds, stipe and rachis without scurf (squamellae and hairs), pinnae and pinnulae with longer pedicle, less segments per pinnulae and evanescent indusia.
\end{abstract}

Keywords: Arborescent ferns, Cyathea antioquiensis, Cyathea incognita, Cyathea longipetiolulata, Neotropics.

\section{Introducción}

Cyathea Sm. fue descrito en 1793, utilizando como tipo a C. arborea (L.) Sm., desde entonces el género ha sufrido la inclusión o exclusión de diversos grupos, entre algunos de los más recientes están la inclusión de los géneros Cnemidaria C. Presl, Hemitelia R. Brown, Trichipteris C. Presl (Crabbe et al. 1975) e Hymenophyllopsis K.I. Goebel (Christenhusz 2009). Así como estudios moleculares recientes favorecen el reconocimiento del género Gymnosphaera Blume, también se ha encontrado que las Cyathea del viejo mundo forman un clado aparte de las neotropicales
(Korall et al. 2007).

Cyathea difiere de otros helechos arborescentes no cyatheoides por tener el tronco y la base de los pecíolos recubiertos de escamas (no de pelos) y por los soros abaxiales redondos (Hoshisaki y Moran 2001). También se diferencia de Alsophila R. Br. por los pecíolos sin espinas negras y las escamas del pecíolo sin setas negras largas; a la vez difiere de Sphaeropteris Bernh. por las escamas del pecíolo comúnmente con células marginales perpendiculares a las centrales y generalmente sin dientes negros verdaderos (Moran 1995).

Murillo et al. (2008) registran para Colombia 73

Universidad Nacional de Costa Rica, Heredia, Costa Rica. e-mail: alfrojasa@yahoo.com

School for Field Studies, Beverly, Perú.

Fecha recepción: Diciembre 10, 2015

Fecha aprobación: Abril 6, 2016

Editor Asociado: Quinto-Mosquera H

(C) Rev. Biodivers. Neotrop. 2016; 6 (2): 121-25 
especies del género Cyathea, siendo este el país que mayor diversidad presenta en este género. En esta investigación se describen tres especies más.

\section{Metodología}

Las especies aquí consideradas son el resultado de la revisión de material en los herbarios Universidad de Antioquia (HUA), Universidad de Caldas (FAUC) y New York Botanical Garden (NY); de la consulta de tipos en la página JSTOR (http://plants.jstor.org/) y de la consulta en floras de helechos (Lehnert 2006a, Murillo et al. 2008, Tryon 1986, Tryon y Stolze 1989) y artículos del género Cyathea (Lehnert 2003, 2006b, 2009, Moran 1991, 1995, Murillo y Murillo 2003, Rojas 2001, 2005, 2007).

\section{Resultados}

Cyathea antioquensis A. Rojas, sp. nov. (Fg. 1). TIPO. COLOMBIA. Antioquia: Mpio. Carmen de Viboral, vereda La Viborita, $6 \mathrm{~km}$ SE del Mpio. de La Unión, en la vía de la troncal Sonsón-La Unión a la vereda Viborita, cabeceras del Río Buey, $5^{\circ} 52^{\prime} \mathrm{N}$, $75^{\circ} 20^{\prime} \mathrm{W}, 2100-2600 \mathrm{~m}, 20$ jun 1991, $R$. Callejas et al. 10196 (holotipo: HUA).

Diagnosis. The new species differs from $C$. latevagans (Baker) Domin by its rhizome scales with a narrower pale margin, stipe and rachis with scurf, and sessile, linear-oblong, falcate pinnae.

Descripción. Epífita; rizoma ca. $1 \mathrm{~cm}$ de diámetro, reptante a suberecto; escamas del rizoma 2-3 x 1-1,5 $\mathrm{mm}$, ovadas, negras con un margen pardo amarillento de ca. $0.1 \mathrm{~mm}$ de ancho, adpresas, imbricadas; frondas 62-85 m de largo; pecíolo 8-18,5 cm de largo, 0,4-0,5 $\mathrm{cm}$ de diámetro basalmente, liso o apenas con botones en la unión de las escamas, negro, opaco, escamoso y con caspilla, las escamas como las del rizoma, densas basalmente y escaseando hasta la mitad, la caspilla de escuámulas adpresas, estas menos $0,5 \mathrm{~mm}$ de largo, medianamente densas; lámina 50-63 x 8-12 $\mathrm{cm}, 1$-pinnado-pinnatífida a 2-pinnada basalmente, la base truncada a levemente reducida, el ápice gradualmente atenuado y pinnatífido; raquis negro en la base y parte media a pardo distalmente, liso, con caspilla ventralmente, pelosos dorsalmente, los pelos 1-1,5 mm de largo, pardos crespos, arqueadas hacia el ápice; pinnas basales y medias 5.5-8 x 1,2-1,5 cm, linear-lanceoladas a linear oblongas, falcadas, sésiles basalmente, atenuadas distalmente; costas pardo amarillentas a pardas, con nódulos basalmente, pelosas dorsalmente, los pelos como los del raquis, dispersamente escamosas ventralmente, las escamas 0,5-2 x 0,2-0,5 mm, pardo amarillentas, bicolores con el centro más oscuro, margen eroso a ciliado; pínnulas o segmentos $0,5-0,8 \times 0,25-0,4 \mathrm{~cm}, 12-15$ pares, sésiles, falcados, obtusos distalmente; nervaduras 5-7 pares por segmento, libres o raramente 1-bifurcadas a pinnadas, glabras dorsalmente, con caspilla amarillenta dispersa dorsalmente; tejido laminar entre las nervaduras glabro en ambas superficies; soros inframedios; sin paráfisos; indusio globoso, pardo-amarillento, que rodea casi completamente al receptáculo.

Etimología. El nombre del epíteto específico hace referencia a la localidad tipo.

Cyathea incognita A. Rojas, sp. nov. (Fg. 2).

TIPO: COLOMBIA. Meta: Mpio. San Juanito, Cañón del Río Guatiquía, near casa San José, $4^{\circ} 30.962^{\prime} \mathrm{N}, 73^{\circ} 41.361^{\prime} \mathrm{W}, 3325 \mathrm{~m}, 3$ May 2007, $M$. Sundue y A. Vasco 1326 (holotipo: HUA; isotipo: NY).

Diagnosis. The new species differs from C. gracilis Griseb by its terrestrial habit, thinner rhizome, rhizome scales with a broad pale margin, shorter and bipinnate fronds, atropurpureous rachis, sessile pinnae and sori without indusia.

Descripción. Terrestre; Rizoma 4-6 mm de diámetro, corto reptante o suberecto; frondas $0,65-0,95 \mathrm{~m}$ de largo; pecíolo 35-50 cm de largo, 1,5-3 mm de diámetro basalmente, atropurpúreo a negro, liso, lustroso; escamas del pecíolo 2,5-4 x 1-1,5 mm lanceoladas, pardo oscuro en el centro, con el margen pardo-amarillento; caspilla ausente; lámina 2-pinnada, el ápice gradualmente atenuado; raquis atropurpúreo, liso, glabro en ambas superficies; pinnas sésiles; pinnas medias 6-8 x 1,6-2,4 cm, lanceoladas, inequiláteras, articuladas basalmente, más anchas del lado basiscópico, sésiles, ápice agudo; costas pajizas; pínnulas 0,8-1,5 x (0,2-) 0,3-0,5 cm, lanceoladas a oblongas, sésiles, margen entero a levemente lobulado, las basales acroscópicas más reducidas; costas pajizas, articuladas al raquis, densamente pelosas dorsalmente, los pelos 0,5-1 mm de largo, crespos, patentes, amarillentos, dispersamente pelosas ventralmente, los pelos menos de $0,5 \mathrm{~mm}$ hialinos a amarillentos, patentes; cóstulas ventralmente con pelos $0,2-0,7 \mathrm{~mm}$, 
hialinos a amarillentos, adpresos, dispersos; venas 6-9 pares por pínnula, libres, simples, pelosas en ambas superficies, los pelos del haz más largos; tejido laminar peloso dorsalmente, glabro ventralmente; escamas costales $0,5-1 \mathrm{~mm} \times 0,3-0,5 \mathrm{~mm}$, lanceoladas pardas con margen amarillento, margen entero a eroso, soros mediales, sin indusio.

Etimología. El nombre del epíteto específico hace referencia a que al ser una planta pequeña con grado de división menor del usual, la misma fue identificada por los colectores como un Thelypteris, pasando a estar escondida (incógnita) para los estudiosos de los helechos arborescentes.

Cyathea longipetiolulata A. Rojas y A. Tejedor, sp. nov. (Fg. 3).

TIPO: COLOMBIA. Risaralda: Municipio de Pueblo Rico, Parque Regional Karagabi, Hoyo Frío, 2500 m, 6 abr 2004, D. Sanín 409 (holotipo: FAUC).

Diagnosis. The new species differs from $C$. divergens Kunze by its ascending fronds, stipe and rachis without scurf (squamellae and hairs), pinnae and pinnulae with longer pedicle, less segments per pinnulae and evanescent indusia.

Descripción. Tronco ca. $2 \mathrm{~m}$ de alto; frondas 3-5 $\mathrm{m}$ de largo, marcadamente ascendentes; pecíolo 140 $\mathrm{cm}$, inerme en casi toda su longitud pero basalmente espinoso, rojo-pajizo opaco en material de herbario, verde brillante en vida, sin escuámulas; escamas del pecíolo 10-20 x 3-11 mm, ovadas a linear lanceoladas, pardo oscuro a pardo-rojizas, concolores a levemente más claras en el margen, peltadas; caspilla ausente; lámina 3-pinnado-lobulada, con las cóstula alada en las pínulas basales, el ápice gradualmente atenuado; raquis pardo a atropurpúreo, liso; pinnas 8-9 pares, pediculadas, el pedículo de $5-10 \mathrm{~cm}$ de largo; pinnas medias 50-75 x 30-35 cm; costas similares al raquis; pínnulas 8-17 x 4-6,5 cm, lanceoladas, pediculadas, el pedículo de 1-2,3 cm; segmentos 1-4 x 0,6-0,9 mm, 10-15 pares, serrados a lobulado-dentados, agudos a obtusos; segmentos basales libres; cóstulas glabras, opacas; nervaduras $10-15$ pares por segmento, 1-2 bifurcadas, glabras; tejido laminar entre las nervaduras glabro; soros costales; paráfisos más cortos que los esporangios, inconspicuos; indusio globoso, evanescente y frecuentemente fragmentado en soros maduros, pardo.

Etimología. El nombre del epíteto específico hace referencia a las pínnulas con largos pedúnculos.

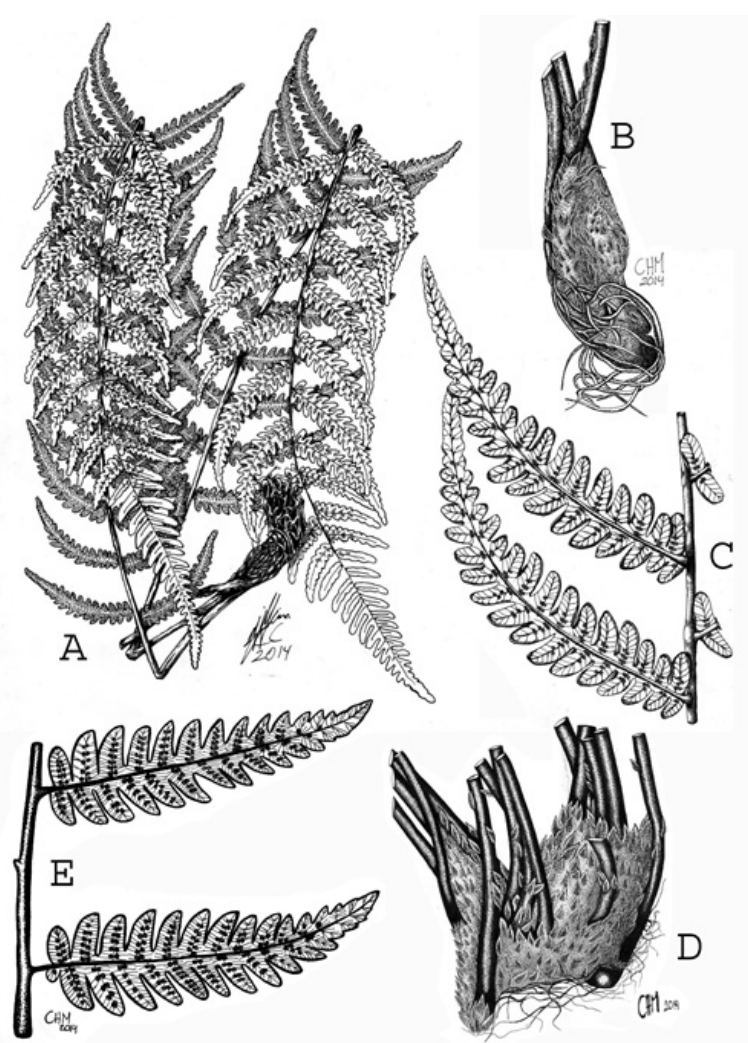

Figura 1. A-C. Cyathea antioquiensis (R. Callejas et al. 10196, HUA). A. Aspecto general del espécimen tipo. B. Detalle del rizoma. C. Pinnas. D-E. Cyathea latevagans $(A$. Arbeláez et al. 82, HUA). D. Detalle del rizoma. E. Pinnas.

Material adicional revisado. COLOMBIA. Risaralda: Montebello, $5.288^{\circ} \mathrm{N}, 76.088^{\circ} \mathrm{W}, 2500 \mathrm{~m}, 13$ jul 2015, F. Giraldo 3134 (BONN, HUA).

\section{Discusión}

Cyathea antioquiensis difiere de C. latevagans por sus escamas del rizoma adpresas e imbricadas ( $v s$. medianamente patentes), con un margen pálido más delgado (margen de ca. $0.1 \mathrm{~mm}$ de ancho vs. 0,2-0,4 $\mathrm{mm}$ ), pecíolo y raquis con caspilla ( $v s$. sin caspillas), pinnas linear-oblongas ( $v s$. deltadas a lanceoladas), falcadas ( $v s$. rectas) y estas sésiles (vs. pediculadas) (Figura 1).

Cyathea incognita difiere de C. gracilis por su hábitat terrestre ( $v s$. generalmente epífita), rizoma más delgado $(0,4-0,6 \mathrm{~cm}$ de diámetro de diámetro vs. 3-8 $\mathrm{cm}$ ), escamas del rizoma con un ancho margen pálido (más de la mitad de su ancho vs. menos de la cuarta parte del ancho), frondas más cortas (0,65-0,95 m de largo vs. (1,5-) 2-3 m), pecíolo más delgado (1,5-3 


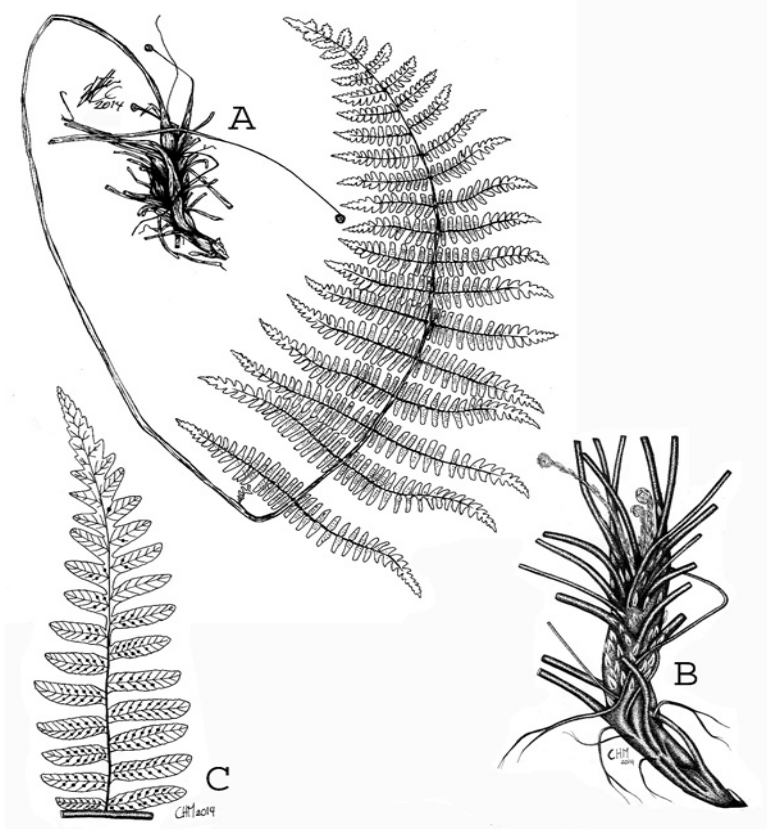

Figura 2. A-C. Cyathea incognita (M. Sundue y A. Vasco 1326, HUA). A. Aspecto general del espécimen tipo. B. Detalle del rizoma. C. Pinna.

mm de diámetro vs. 1,5-3 cm), lámina 2-pinnada ( $v s$. 2-pinnado-pinnatífida a 3-pinnado-lobulada), raquis atropurpúreo (vs. pardo claro a pardo oscuro), pinnas sésiles ( $v s$. pediculadas) y soros sin indusio ( $v s$. indusiado) (Figura 2).

Cyathea longipetiolulata difiere de $C$. divergens por sus frondas ascendentes ( $v s$. arqueadas o pendulares), pecíolo y raquis sin caspa de escuámulas y pelos ( $v s$. con caspa presente), pinnas y pínnulas con pedículos más largos (pinnas con pedículo de $5-10 \mathrm{~cm}$ de largo vs. 2-6 cm, pínnulas con pedículo de 1-2,3 $\mathrm{cm} v s .0,2-1 \mathrm{~cm})$, menos segmentos por pínnula (1015 pares $v s .15-20$ pares) e indusio evanescente ( $v s$. persistente). También difiere de Cyathea gracilis por su hábitat terrestre (vs. generalmente epífito); rizoma hasta $2 \mathrm{~m}$ de alto ( $v s$. compacto o hasta $0,5 \mathrm{~m}$ de alto), frondas ascendentes (vs. arqueadas o pendulares), pinnas y pínnulas con pedículos más largos (pinnas con pedículo de $5-10 \mathrm{~cm}$ de largo vs. $2-5 \mathrm{~cm}$, pínnulas con pedículo de $1-2,3 \mathrm{~cm} v s .0,4-0,8 \mathrm{~cm})$ e indusio evanescente (vs. persistente) (Figura 3).

Cyathea longipetiolulata se encontró entre $2250 \mathrm{y}$ $2500 \mathrm{~m}$ de elevación, en bosque húmedo montano con gran abundancia de musgos y epifitas. En la mayoría de los casos se observó creciendo dentro del bosque

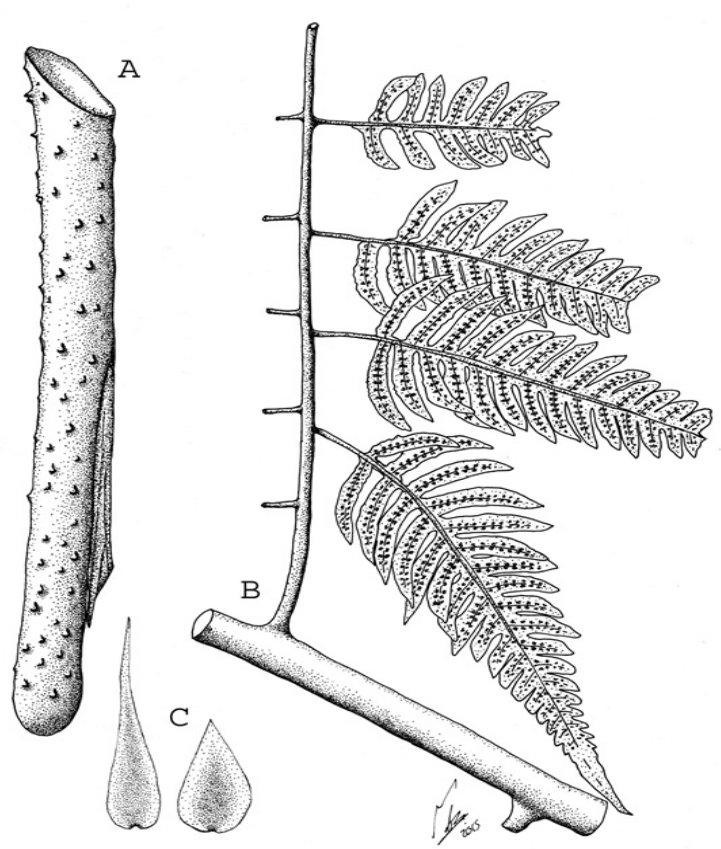

Figura 3. A-C. Cyathea longipetiolulata (D. Sanín 409, FAUC). A. Detalle del pecíolo. B. Detalle de la lámina. C. Escama del pecíolo.

y más raramente expuesta al borde de carreteras. En ambos hábitats, las frondas se disponían de forma ascendente, y se arqueaban solo distalmente, lo cual diferencia a $C$. longipetiolulata de especies potencialmente confundibles como $C$. divergens y $C$. gracilis, en las que las frondas son pendulares o arqueadas. A pesar del tronco relativamente corto (hasta $2 \mathrm{~m}$ ) de C. longipetiolulata, sus frondas largas (hasta 5 m) de pecíolos casi verticales alcanzan el dosel del bosque, confiriéndole a esta especie un hábito y nicho ecológico probablemente único entre los helechos arborescentes Neotropicales (Figura 4).

\section{Agradecimientos}

Se agradece a los herbarios University of Bonn (BONN), Universidad de Caldas (FAUC), Universidad de Antioquia (HUA) y New York Botanical Garden (NY) por permitirnos consultar sus colecciones. A Christian Herrera Martínez, William Calderón Campos y Pablo Muñoz Cambronero por sus excelentes ilustraciones. A los revisores anónimos que contribuyeron con su experiencia a mejorar este artículo. 


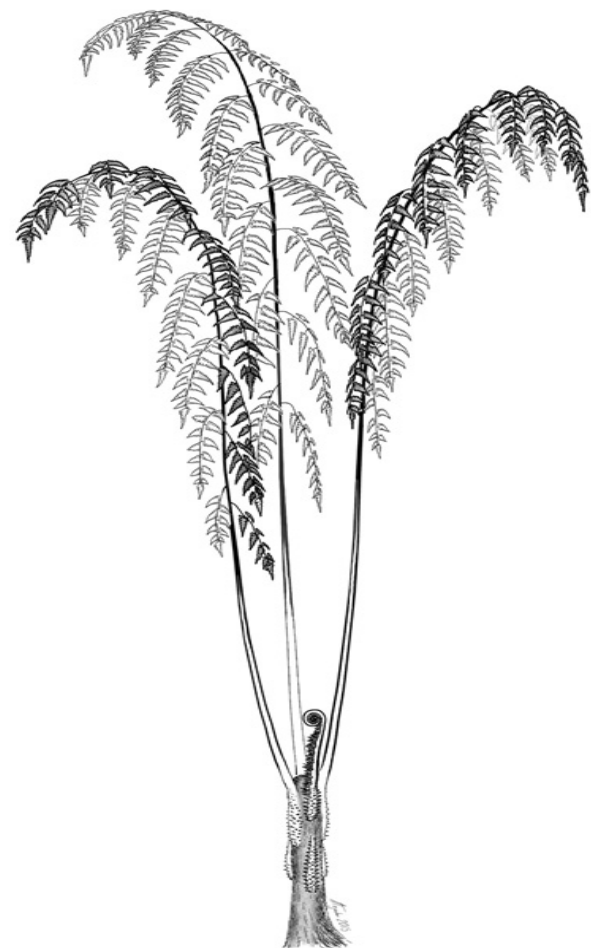

Figura 4. Hábito de Cyathea longipetiolulata (F. Giraldo 3134 (HUA).

\section{Literatura citada}

Crabbe JA, Jermy AC, Mickel JT. 1975. A new generic sequence for the pteridophyte herbarium. Fern Gazette 11 (2-3): 141-62.

Christenhusz MJM. 2009. New combinations and an overview of Cyathea subg. Hymenophyllopsis (Cyatheaceae). Phytotaxa 1: 37-42.
Hoshisaki BJ, Moran RC. 2001. Fern Grower's Manual. Portland: Timber Press; 604 pp.

Korall P, Conant DS, Metzgar JS, Schneider H, Pryer KM. 2007. A molecular phylogeny of scaly tree ferns (Cyatheaceae). Am J Bot. 94 (5): 873-86.

Lehnert M. 2003. Six new tree ferns from the Andes. Am Fern J. 93: 169-83.

Lehnert M. 2006a. New species and records of tree ferns (Cyatheaceae, Pteridophyta) from the northern Andes. Organisms, Diversity \& Evolution. 6 (4): 321-2. doi:10.1016/j. ode.2005.12.002

Lehnert M. 2006b. The Cyatheaceae and Dicksoniaceae (Pteridophyta) of Bolivia. Brittonia. 58: 229-44.

Lehnert M. 2009. Three new species of scaly tree ferns $(C y$ athea-Cyatheaceae) from the northern Andes. Phytotaxa. 1: 43-56.

Moran RC. 1991. Eight new species of three ferns (Cyathea, Cyatheaceae) from the American tropics and tree new combinations. Novon. 1: 88-104.

Moran RC. 1995. Five new species and two new combinations of ferns (Polypodiopsida) from Ecuador. Nordic J Bot. 15 (1): 49-58.

Murillo J, Murillo MT. 2003. Pteridófitos de Colombia IV. Novedades en Cyathea (Cyatheaceae). Rev Acad Colomb Cien. 27: 45-51.

Murillo MT, Murillo J, León A, Triana-Moreno LA. 2008. Los pteridófitos de Colombia. Bogotá : Arfo, 533 pp.

Rojas AF. 2001. Nuevas especies, nombres nuevamente utilizados y nuevas distribuciones en los helechos arborescentes (Filicales: Cyatheaceae) para el neotrópico. Rev BiolTrop. 49: 453-66.

Rojas AF. 2005. Nuevos taxa en helechos arborescentes (Filicales: Cyatheaceae) en Costa Rica. Lankesteriana 5: 191-200.

Rojas AF. 2007. Novedades en Cyathea (Filicales: Cyatheaceae) para Costa Rica y Panamá. MES. 2 (3): 1-13.

Tryon RM. 1986. Flora of Ecuador $N^{\circ} 27$. 13. Cyatheaceae. 59 pp. Tryon RM, Stolze RG. 1989. Pteridophyta of Peru. Part I. 1.Ophioglossaceae-12.Cyatheaceae. Fieldiana Bot.20: 145. 